\section{Gold Nanoparticles Enhance Clarity of Latent Fingerprints}

In the 1941 classic film The Maltese Falcon, detective Sam Spade finds out the hard way just how dangerous gold can be-but he never suspects that one day gold might help him do his job better. In an article published in a recent issue of Chemical Communications (p. 1142; DOI: 10.1039/b618966k), M. Sametband, I. Shweky, U. Banin, D. Mandler, and J. Almog of The Hebrew University in Jerusalem, Israel, demonstrate a method of using nanoparticles of gold to significantly enhance the intensity and clarity of latent (invisible) fingerprints.

The standard method for capturing latent fingerprints on wet porous surfaces has not changed in decades. Silver physical developer (Ag-PD) is applied to the surface, and the fatty components of the fingerprint residue (sebum) catalyze the deposition of metallic silver, leaving a black residue along the fingerprint ridges. However, Ag-PD is not very stable, and is not always effective at developing faint prints. Previous work has shown that gold nanoparticles applied to latent fingerprints can enhance Ag-PD deposition through an ionic interaction, in a process known as multi-metal deposition (MMD). Sametband and colleagues, inspired by this earlier work, hypothesized that hydrophobic interactions could lead to the adsorption of gold nanoparticles onto sebum-rich fingerprint ridges, and that these nanoparticles would then catalyze the silver reduction from Ag-PD, enhancing the fingerprint images.

To test this idea, the researchers began with a petroleum ether solution of gold nanoparticles (Au-NPs), which were stabilized with alkanethiol chains (i.e., octadecanethiol, tetradecanethiol, or decanethiol) at different concentrations. The spherical nanoparticles were $2-3 \mathrm{~nm}$ in diameter, roughly five times smaller than the nanoparticles used in the MMD method. The researchers next produced sebum-rich fingerprint samples on paper strips, and immersed them in the solution for times ranging from $10 \mathrm{~s}$ to $5 \mathrm{~min}$, after which they applied Ag-PD. They found that the fingerprint impressions were significantly stronger and clearer than they were for samples without the Au-NP treatment, with optimal results using a $0.04 \%(\mathrm{w} / \mathrm{v})$ solution and $3 \mathrm{~min}$ of immersion time. Samples without the Au-NP treatment took 10-15 min to develop. The method also worked for sebum-rich fingerprints on silicon surfaces, which are not porous. The Au-NPs stabilized with octadecanethiol aggregated better than the Au-NPs stabilized with tetradecanethiol which in turn aggregated better than the Au-NPs stabilized with decanethiol. In a final set of experiments, the researchers examined the effect of $\mathrm{CdSe} / \mathrm{ZnS}$ nanoparticles on latent fingerprints, and found that aggregates of the nanoparticles adhered to the fingerprint ridges and were clearly visible under ultraviolet illumination, without the need for further chemical enhancement. In light of these results, nanoparticles may someday become a standard part of the forensic toolkit, and the Sam Spades of the future may think that gold is just as useful as it is dangerous.

\section{COLIN MCCORMICK}

\section{Suspended Microfluidic Channels Can Weigh Biomolecules and Single Nanoparticles in Fluid Media}

Nanomechanical resonators have enabled the measurement of masses as light as 7 zeptograms ( $1 \mathrm{zg}=10^{-21} \mathrm{~g}$; i. e., the mass of $\sim 4185$ protons) in vacuum. In order to resolve such small mass changes, extremely light resonators ringing at very pure tones are required. However, such resonators are severely degraded by viscosity, preventing applications in fluids. T.P. Burg, S.R. Manalis, and co-workers from the Massachusetts Institute of Technology, W. Shen and co-workers from Innovative Micro Technology, and K. Babcock from Affinity Biosensors, in Santa Barbara, California demonstrated, in the April 26 issue of Nature (p. 1066; DOI: 10.1038 /nature05741), that suspended microchannel resonators in which viscous loss due to the fluid can be neglected with respect to the intrinsic damping of the silicon crystal resonator can weigh single nanoparticles, single bacterial cells, and sub-monolayers of adsorbed proteins in water with sub-femtogram resolution. This result achieved a mass resolution in solution of 300 attograms ( $\left.1 \mathrm{ag}=10^{-18} \mathrm{~g}\right)$ an improvement of six orders of magnitude over quartz crystal microbalances (mass resolution $\sim 1 \mathrm{ng}$ ).

The researchers fabricated suspended microfluidic channels with walls $2-3 \mu \mathrm{m}$ thick and a $3-\mu \mathrm{m}$ fluid layer in cantilevershaped resonators by forming buried channels in silicon-on-insulator wafers, followed by wafer thinning and dry etching. They integrated an electrostatic drive electrode under the cantilevers and detected the cantilever vibration optically. The resonance frequency of such suspended microchannels is highly sensitive to the presence of molecules or particles whose mass density differs from that of the solution, and that are absorbed on the surface of the functionalized channels, or suspended in a solution flowing through the resonator-a unique feature of this device.

The researchers measured the binding of goat anti-mouse immunoglobulin- $\gamma$ molecules to anti-goat immunoglobulin- $\gamma$ antibodies that were immobilized on the channel walls functionalized with a layer of Neutravidin bound to poly(ethyleneglycol)biotin grafted poly-L-lysine. The 